\title{
Apuntes sobre encuestas electorales
}

\author{
Notes about election polls
}

\section{INTRODUCCIÓN}

Las encuestas preelectorales suelen despertar gran interés por el valor predictivo que se les puede atribuir o que puedan tener, y por el efecto que puedan generar en los votantes. También se usan como herramienta efectiva para el manejo de las campañas de los candidatos. Existen tres tipos de encuestas electorales: preelectorales, boca de urna y postelectorales, en cada caso cumplen funciones diferentes en relación con los procesos electorales. Philippe Maarek (1) considera que la publicación de encuestas preelectorales influye en la decisión de voto mediante efectos directos e indirectos.

Es en coyunturas preelectorales cuando las encuestas suelen concitar la mayor atención, especialmente por el valor predictivo que se les puede atribuir o que puedan tener, y por el efecto que puedan generar en los votantes, de manera especial en los indecisos, que constituyen un porcentaje usualmente muy alto durante casi toda la etapa preelectoral. Esto hace que las encuestas sean controvertidas y discutidas en el mundo.

En los procesos electorales, las encuestas también son utilizadas como una herramienta efectiva para el manejo eficiente de las campañas de los candidatos, asumiendo de esta manera un papel protagónico y permanente en las campañas.

Además de sus implicancias electorales, las encuestas tienen asimismo presencia en la vida política institucional en épocas no electorales.

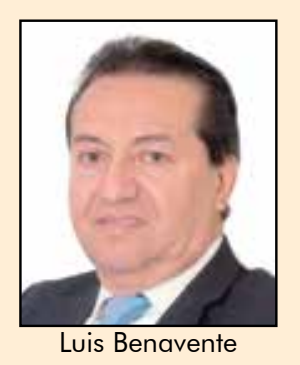

luis.benavente@voxpopuli.pe

Actualmente son utilizadas para conocer la opinión de los ciudadanos acerca de diversos temas políticos, económicos y sociales. Las encuestadoras realizan sondeos periódicos sobre todos estos aspectos que son difundidos a través de los medios de comunicación, alimentando de este modo el debate político.

En la actualidad, las encuestas de opinión son una práctica habitual en todos los países con regímenes democráticos, constituyéndose en un instrumento de medición de la opinión de los ciudadanos.

1 Licenciado en Ciencias de la Comunicación y magíster en Administración, graduado por la Universidad de Lima. Actualmente se desempeña como director de Vox Populi Comunicación y Marketing, y profesor principal del Máster en Comunicación Política e Institucional del Instituto Universitario Ortega y Gasset de Madrid, y del Máster en Campañas Electorales del Jurado Nacional de Elecciones. Fue director del Grupo de Opinión Pública de la Universidad de Lima (1994-2002). 


\section{Historia de las encuestas de opinión}

Desde la aparición de la primera encuesta preelectoral en el diario Harrisgurg Pennsylvanian en 1824 con motivo de la elección presidencial en los Estados Unidos, muchísimo es lo que se ha hecho y dicho en relación con las encuestas de opinión. Sin embargo, es a partir de la cuarta década del siglo pasado que las encuestas recién cuentan con el rigor del método muestral, que de manera accidental descubrió George Gallup, un periodista y matemático -rara y potente combinación- de 35 años que fundó el Instituto Americano de Opinión Pública -luego se Ilamaría simplemente Gallup- en los Estados Unidos.

En 1936 Gallup utilizó su flamante método muestral y entrevistó solo a 3000 ciudadanos y pronosticó que Roosevelt obtendría el $56 \%$ de los votos y ganaría la elección.

Literary Digest había enviado el cuestionario a nada menos que 2 millones 300 mil votantes -una cantidad suficientemente grande como para asegurarse un resultado confiable, independientemente del número de ciudadanos que respondiera- y declaró que Alfred Landon del Partido Republicano ganaría la elección al demócrata Franklin D. Roosevelt y sería el nuevo presidente de los Estados Unidos. El sondeo dio solo 42\% de los votos a Roosevelt.

Concluido el conteo oficial, Roosevelt ganó con el $61 \%$ de los votos. Gallup y su método muestral también ganaron. Literary Digest y su método no científico perdieron. Poco después dejó de funcionar.

George Gallup había descubierto la técnica del muestreo de manera casual. Una mañana estaba en una granja, metió la mano a un saco y recogió un puñado de granos blancos y negros, los contó, 42 eran blancos y 20 negros. Hizo esto varias veces y cada vez que sacaba un puñado de granos encontraba una proporción de granos muy parecida.
Luego se supo que los sacos contenían granos en una proporción de dos a uno. Gallup concluyó que si una muestra representa a un todo, lo reproducirá siempre. Buscó extrapolar su experiencia a la población nacional de los Estados Unidos. La muestra debía reproducir la misma proporción por sexo, clase social, raza, etcétera, al universo de ciudadanos del país.

En 1945 la sucursal de Gallup en el Reino Unido pronosticó el triunfo de Clement Attlee del Partido Laborista, quien fue elegido primer ministro al vencer a Winston Churchill del Partido Conservador. El sondeo de Gallup fue contra la corriente, pues Churchill era considerado fijo por la gran simpatía que generaba.

En la campaña presidencial de 1952 en los Estados Unidos, los republicanos realizaron por primera vez encuestas de opinión para determinar expectativas de los ciudadanos y seleccionar temas de campaña para 49 spots televisivos para la candidatura de "Ike" Eisenhower.

En la segunda mitad del siglo veinte, las encuestas se desarrollaron gradualmente en todas las democracias del mundo y se convirtieron en parte importante de las campañas electorales y del sistema político mismo. Han pasado 77 años desde aquel sondeo muestral en los Estados Unidos y hoy las encuestas son una realidad, una polémica realidad.

\section{Encuestas de opinión}

Todas las personas tenemos actitudes, opiniones y conductas en relación con lo que ocurre en muchos aspectos de nuestro entorno, y la estructura de esas actitudes, opiniones y conductas individuales da como resultado el surgimiento de actitudes, opiniones y conductas colectivas.

Los investigadores y los estrategas políticos y sociales obtienen información sobre temas políticos, sociales, económicos, empresariales, culturales, etcétera, mediante el uso de técnicas de investigación, 
entre ellas las encuestas de opinión pública.

Los estudios de opinión pública surgieron de la inquietud y necesidad de conocer cómo las personas perciben muchas cosas de la realidad. Así aparecieron las mediciones de opinión pública mediante encuestas, primero con el uso de procedimientos no aleatorios y posteriormente mediante el uso del método cuantitativo del muestreo.

Al ser muy cambiante el medio ambiente social, los estudios de opinión pública deben ser tomados de manera referencial, la opinión pública no es estable, en muchas circunstancias puede cambiar rápida y hasta dramáticamente.

\section{Encuestas electorales}

Las encuestas electorales tienen como objeto los procesos electorales y se realizan antes, durante y después de las elecciones. Se clasifican en tres categorías de acuerdo con el momento en que se realizan y tienen distintos propósitos: encuestas preelectorales, encuestas a boca de urna, encuestas postelectorales.

\section{Encuestas preelectorales}

Tienen una gran importancia pública porque miden la intención de voto y las variaciones de las preferencias por los distintos candidatos durante la etapa preelectoral, generan muchas expectativas en los medios de comunicación, partidos políticos y ciudadanos que pueden ver en estas encuestas un pronóstico de los resultados electorales.

Las encuestas preelectorales también permiten conocer las actitudes políticas y electorales de los votantes y cómo van variando en el tiempo. Esta información es muy valiosa para la prensa, los ciudadanos, los investigadores y los estrategas de campaña.

Permiten medir el clima de opinión de la etapa preelectoral, en el que interviene la acción de los candidatos y sus partidos a través de comportamientos y mensajes públicos orientados a modificar dicho clima de opinión, así como las campañas negativas que igualmente tienen alto impacto en el devenir de la campaña, la publicidad en medios que también busca persuadir a los votantes, la acción en las redes sociales que ha generado un espacio cada vez más amplio de participación de los ciudadanos mediante opiniones y acceso a la información, los mensajes de la prensa que obviamente intervienen en el proceso de formación de la opinión pública electoral.

Estas encuestas permiten además evaluar a los candidatos, sus atributos, fortalezas y debilidades, su comportamiento preelectoral, sus comunicaciones, las actitudes de distintos segmentos de votantes hacia los candidatos, la transferencia de votos en caso de balotajes, etcétera, un cúmulo de información para manejo de toda aquella persona y organización que esté o se considere involucrada en el proceso político de las elecciones que vienen, es decir, para manejo de muchos.

\section{Encuestas a boca de urna}

Tienen muestras muy grandes que deben cubrir todas las áreas geográficas de la circunscripción electoral, el cuestionario es muy breve, las entrevistas son igualmente breves y se realizan a los votantes al momento de salir del local de votación (exit poll) para que confiesen a qué candidato acaban de votar. Si las normas electorales lo admiten, estas encuestas permiten tener resultados ponderados muestrales antes del término de la jornada de votación. De esta manera, al momento de concluir oficialmente la votación, los medios de comunicación disponen de resultados estadísticos no oficiales que comunican públicamente ante una expectativa muy alta de los ciudadanos. Gracias a las telecomunicaciones, estos resultados podrían llegar a ser conocidos simultáneamente en todo el planeta.

\section{Inconvenientes de las encuestas a boca de urna:}

El reporte de la cuota tomada por cada 
entrevistador, la organización de la información y el tratamiento de datos, son tres procesos que aun en el caso de ser muy eficientes, demandan tiempos mínimos que condicionan que el trabajo de campo concluya unas horas antes del término oficial de la votación, de manera que la encuesta a boca de urna no registra la confesión de voto de quienes votaron en ese horario, el mismo que resulta fuera de la muestra.

Por distintas razones (vergüenza, temor) hay entrevistados que aceptan la entrevista pero que mienten al confesar su voto, de modo que ese porcentaje se suma al margen de error estadístico y da como resultado una mesas de votación en una circunscripción electoral. Dichas actas suelen ser publicadas en la puerta del local de votación, a donde acude el representante de la institución - empresa encuestadora para recoger la información y reportarla rápidamente, para lo cual dispone de un eficiente sistema multimodal de comunicaciones con la central de campo y tratamiento de datos.

El diseño de la muestra se hace con el uso de la técnica del muestreo sistemático, que mediante un salteador sistemático permite determinar con exactitud las mesas seleccionadas.

El uso de esta modalidad ha permitido

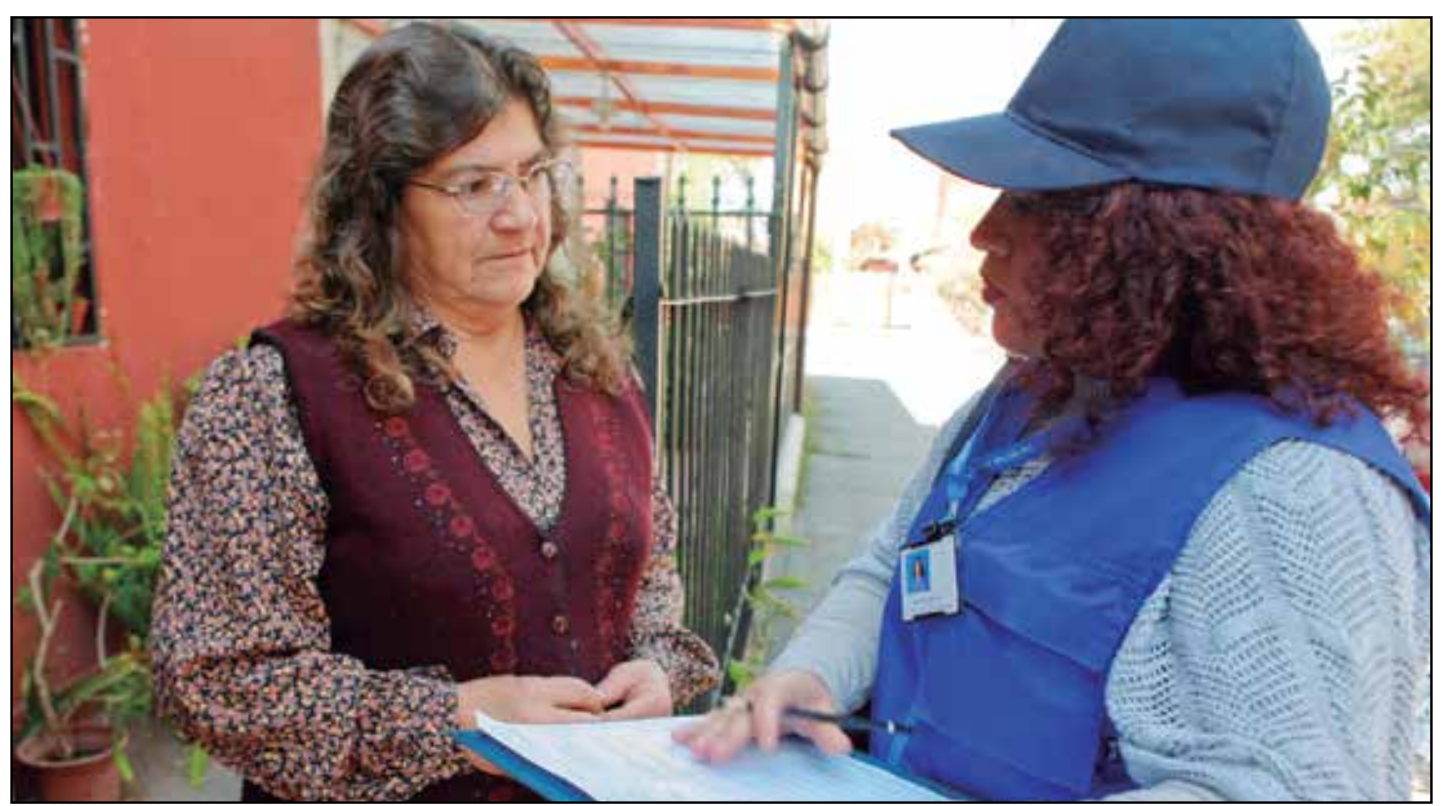

Figura $\mathrm{N}^{\circ}$ 1: Encuestas electorales.

Fuente: Diario El Tipógrafo: http://eltipografo.cl

distorsión que emerge en el momento de comparar la encuesta a boca de urna con el resultado oficial.

\section{Encuestas postelectorales}

Existen dos tipos de estudios cuantitativos que suelen realizarse después de las elecciones:

\section{Conteo rápido:}

El conteo rápido consiste en la toma de información de los resultados que contienen las actas de escrutinio de una muestra de las obtener resultados de muy alta precisión cuando han sido comparados con resultados oficiales en elecciones realizadas en muchos países del mundo.

De esta manera se dispone de resultados postelectorales no oficiales mediante conteo rápido de muy alta calidad y confiabilidad que pueden ser comparados con los resultados al concluir la cuenta oficial.

\section{Evaluaciones postelectorales:}

Se realizan poco tiempo después del día de 
la elección, como puede ser una semana después, y permiten obtener información muy confiable sobre opiniones, actitudes, motivaciones y comportamientos electorales, como por ejemplo determinar por qué el ciudadano asistió a votar, por qué votó a un candidato o candidata, por qué no votó a tal candidato o candidata, cuándo decidió a quién votar, a quién votaron las personas de su entorno más cercano, qué actitud tuvo hacia el proceso electoral, qué expectativas tiene en el candidato elegido, etcétera.

Se considera que esta información es muy valiosa porque se ha obtenido inmediatamente después del proceso electoral y porque puede orientar el análisis de futuras elecciones y ser utilizada por los estrategas electorales para el diseño de su planes. Al ser claro que los fenómenos sociales y políticos no son fácilmente replicables porque las condiciones en las que se producen son cambiantes, se considera que los estudios postelectorales muestran actitudes y conductas que no se van a repetir de manera mecánica.

\section{Dificultades de las encuestas electorales}

Una importante dificultad o limitación que se enfrenta puede ser el tamaño del presupuesto, pues si este no es suficiente surge la tentación inmediata de reducir el tamaño de la muestra para reducir los costos, especialmente los costos directos. En el caso de Perú, esto ha llevado que muchas veces se realicen encuestas sobre elecciones nacionales en los siguientes ámbitos geográficos:

1. Solo en las provincias de Lima y Callao, que juntas representan el $33,39 \%$ de la población electoral peruana. Estas encuestas excluyen de su representatividad al $66,61 \%$ de la población electoral total.

2. Solo en el ámbito urbano nacional, que representa el $75,9 \%$ de la población general peruana y que excluye de su representatividad al $24,1 \%$ de dicha población, que es el porcentaje de población rural nacional (2).

El informe de las encuestas debe contener además una ficha técnica que explique claramente el ámbito geográfico donde fue realizada, pero con la publicación de la encuesta el lector tiende a percibir que los resultados tienen un valor general (o representatividad nacional), a pesar de que se hubiera precisado el ámbito geográfico, el cual a menudo es puesto en "letra chiquita" por los medios de comunicación. Esta es una suerte de "ilusión óptica" o percepción distorsionada.

Esta distorsión es importante y tiene un impacto negativo en la transparencia de los procesos electorales. Por lo tanto, mi sugerencia es que la regulación de encuestas electorales prohíba la publicación de encuestas sobre elecciones nacionales cuyo diseño muestral no considere un determinado porcentaje mínimo de provincias de todo el país, y que esas provincias representen otro determinado porcentaje mínimo de la población electoral rural nacional.

Otras dificultades o limitaciones que enfrentan las instituciones y empresas encuestadoras son las siguientes:

1. La posible falta de cartografía actualizada y adecuada.

2. La posible falta de personal calificado.

3. La posible reducida penetración del teléfono.

4. La posible reducida penetración de Internet.

5. Las dificultades de acceso a zonas apartadas del país.

6. La desconfianza de los ciudadanos.

Efectos de las encuestas electorales en la decisión de voto

De acuerdo con Philippe Maarek (3), las encuestas de opinión tienen efectos directos e indirectos: 


\section{Efectos directos:}

La publicación de los resultados de las encuestas de opinión durante la campaña puede producir modificaciones en la intención de voto de los electores.

Los electores poco movilizados de un candidato con poca intención de voto, al conocer el bajo nivel alcanzado en las encuestas, sienten la necesidad de apoyarlo y le confirman su voto. Otros electores pueden modificar su intención de voto en favor suyo. Éste es el efecto underdog.

También ocurre que la publicación de los resultados de las encuestas puede animar a los electores indecisos a votar por el candidato que esté adelante en las encuestas. De esta manera forman parte de la mayoría y se aseguran un triunfo. Éste es el efecto bandwagon.

Los políticos saben que la publicación de encuestas favorables a sus candidaturas les genera un fuerte efecto bandwagon en los propios militantes y simpatizantes. El resultado favorable sube la moral de los militantes y aumenta el número de simpatizantes.

Los cajeros de las campañas llevan encuestas favorables a los financistas de campañas, como carta de garantía para aumentar las donaciones que esperan recibir.

\section{Efectos indirectos:}

Las encuestas de opinión suelen convertirse en el contenido del debate político, distorsionando el proceso de comunicación política. Muchas veces la discusión no se da en torno al plan de gobierno o las propuestas del candidato, se da alrededor de los resultados de una encuesta.

De los resultados de una encuesta puede hacerse muchos titulares de primera plana, y los periodistas interrogarán a los políticos y a los analistas sobre los resultados de las encuestas.

Conforme aumenta la atención mediática en los números de las encuestas y en las variaciones de estos a través del tiempo, la campaña pierde calidad, los problemas políticos y sociales pasan a un segundo plano o desaparecen.

\section{CONCLUSIONES}

Las encuestas de opinión adquieren particular importancia en coyunturas preelectorales, debido al valor predictivo que se les atribuye o que pueden tener, y por el efecto que pueden generar en los votantes, de manera especial en los indecisos.

Además de sus implicancias electorales, las encuestas también tienen presencia en la vida política institucional en épocas no electorales; son utilizadas para conocer la opinión de los ciudadanos acerca de todo tipo de asuntos políticos, económicos y sociales. Existe especial interés en la evaluación de los gobernantes.

Desde 1936, cuando Gallup utilizó su flamante método muestral en Estados Unidos y entrevistó solo a 3000 ciudadanos y pronosticó que Roosevelt ganaría la elección, hasta la actualidad, las encuestas de opinión han sido y son una práctica habitual en todos los países con regímenes democráticos, constituyéndose en un instrumento de medición de la opinión de los ciudadanos.

Las encuestas de opinión son controvertidas y discutidas en el mundo.

Se considera que la publicación de encuestas electorales influye en la decisión de voto, mediante efectos directos e indirectos.

Efectos directos: Los electores poco movilizados de un candidato con poca intención de voto, al conocer el bajo nivel alcanzado en las encuestas, sienten la necesidad de apoyarlo y le confirman su voto. También ocurre que los electores indecisos a votar al candidato que va adelante en las encuestas, se animan a 
votarlo, para formar parte de la mayoría y compartir un triunfo.

Los políticos saben que la publicación de encuestas favorables a sus candidaturas les genera un fuerte efecto positivo en los propios militantes y simpatizantes, pues el resultado favorable sube la moral de los militantes y aumenta el número de simpatizantes.

Efectos indirectos: Las encuestas de opinión suelen convertirse en el contenido del debate político, distorsionando el proceso de comunicación política. Muchas veces la discusión no se da en torno al plan de gobierno o las propuestas del candidato, se da alrededor de las encuestas.

De los números de las encuestas se hacen muchos titulares en los medios de comunicación, y los periodistas interrogan a políticos y analistas sobre esos resultados. Conforme aumenta la atención mediática en las encuestas, la campaña pierde calidad, los problemas políticos y sociales pasan a un segundo plano o desaparecen.

\section{REFERENCIAS BIBLIOGRÁFICAS}

1. Maarek P. Marketing político y comunicación. Nueva edición. España: Paidós lbérica; 2009.

2. Instituto Nacional de Estadística e Informática. Perú: grado de urbanización 2007 [Internet]. Lima: Instituto Nacional de Estadística e Informática; 2013 [consulta: 29/08/2013]. Disponible en: http://www 1 .inei.gob.pe/ perucifrasHTM/infdem/cuadro. asp? $\operatorname{cod}=11221$ \& name $=$ po09\&ext $=$ if

3. Maarek P. Marketing político y comunicación. Claves para una buena información política. 1ํㅡ. ed. Barcelona: Ediciones Paidós; 2007. 